\title{
ON KOLMOGOROV TYPE INEQUALITIES FOR THE NORMS OF OPERATORS OF INTERMEDIATE DERIVATIVES IN THE SPACE OF SMOOTH VECTOR FUNCTIONS ON A FINITE SEGMENT
}

\author{
HAMIDULLA I. ASLANOV AND GUNEL M. EYVAZLY
}

\begin{abstract}
The paper is devoted to the Kolmogorov type inequalities in some spaces of smooth Sobolev type vector functions between the norms of functions and their derivatives, more exactly, the operators of intermediate derivatives. The obtained estimations play in important role when proving solvability of boundary value problems for operator-differential equations on a finite segment. Sufficient conditions for solvability of boundary value problems are expressed by means of the norm of the operators of intermediate derivatives.
\end{abstract}

\section{Introduction}

First E.Landau and then J.Hadamard paid attention to the inequalities between the norms of functions and their derivatives [12]. Namely, for a twice continuously differentiable function on all axis, an inequality between the norms of a first order derivative and the norms of the function itself and second order derivative was obtained. Further a number of authors have obtained such inequalities. The first more complete result was obtained by A.N.Kolmogorov [13], who proved an exact inequality for $n$-times continuously differentiable functions between the norms of derivatives of order $k=0,1, \ldots, n-1$ and the norm of the function itself and $n$-th order derivative.

Afterwards, a great number of papers were devoted to obtaining similar Kolmogorov inequalities in the space $L_{p}(1 \leq p<\infty)$ on all axis, semi-axis and on a finite segment. See the papers by V.V.Arestov [4], V.N.Gabushin [10], S.B.Stechkin [20], N.P.Kuptsov [14], V.I.Burenkov [6] and others. One can find abstract analogs of these inequalities in the book of J.L.Lions and E.Majenes [15] and in the paper of Yu.I.Lyubich [16].

Let $H$ be a separable Hilbert space, $A$ be a positive-definite self-adjoint operator in $H$. Then it is proved that the space

$$
W_{2}^{m}([a, b] ; H)=\left\{u(t): u^{(m)}(t) \in L_{2}([a, b] ; H), A^{m} u \in L_{2}([a, b] ; H)\right\}
$$

2010 Mathematics Subject Classification. 34C10, 35J40, 47A50, 47D03.

Key words and phrases. Operator, operator-differential equations, boundary conditions, regular solvability, Sobolev space, Hilbert space, norm, scalar product, isomorphism. 
is a Hilbert space with respect to the norm,

$$
\|u\|_{W_{2}^{m}([a, b] ; H)}=\left(\int_{a}^{b}\left(\left\|u^{(m)}(t)\right\|^{2}+\left\|A^{m} u\right\|^{2}\right) d t\right)^{1 / 2}
$$

and we have the inequalities:

$$
\left\|A^{n-j} u^{(j)}\right\|_{L_{2}([a, b] ; H)} \leq C_{n, j}(a, b)\|u\|_{W_{2}^{m}([a, b] ; H)}
$$

$j=0,1,2, \ldots, n-1$. Here, all derivatives are understood in the sense of distribution theory, $-\infty \leq a \leq b \leq \infty$.

The inequalities of the form (1.1) have applications in theory of elliptic boundary value problems [1].

Note that when $H=R$, there is a great interest to such inequalities in connection with their close relation to the problems of the best approximation of a differentiation operator by bounded operators and also the best approximation of a class of functions by other ones (see $[6,10,20])$.

The further interest to finding the exact values of the norms of operators of intermediate derivatives, i.e. finding of the exact constants in inequalities (1.1), is due to the fact that they have important applications in theory of solvability of boundary value problems for operator-differential equations. Mirzoyev S.S. $[17,18]$ was the first who paid attention to these applications.

In these works, it was indicated that finding the exact values of constants in inequalities (1.1) enables to find a wider class of operator-differential equations for which there are solvability theorems.

Important results of theory of operator-differential equations were obtained in the papers of Gasymov M.G. [11], Dubinsky Yu.A. [7], Aliyev A.R. [2], Aliyev V.A. [3], Aslanov G.I. [5], Shkalikov A.A. [19] and others.

\section{Problem statement}

Let $H$ be a separable Hilbert space with a scalar product $(x, y)_{H}$, where $x, y \in$ $H$. Denote by $L_{2}([0,1] ; H)$ a Hilbert space of all vector-functions determined on $[0,1]$ with the values in $H$, that has the norm

$$
\|f\|=\left(\int_{0}^{1}\|f(t)\|_{H}^{2} d t\right)^{1 / 2} .
$$

Let $A$ be a self-adjoint positive-definite operator in $H$ with the domain of definition $D(A)$. The domain of definition of the operator $A^{p}(p \geq 0)$ is a Hilbert space $H_{p}$ with respect to the scalar product $(x, y)_{H_{p}}=\left(A^{p} x, A^{p} y\right)_{H}, x, y \in$ $D\left(A^{p}\right)$. For $p=0$ we assume $H_{0}=H,(x, y)_{H_{0}}=(x, y)_{0}, x, y \in H$.

Let us determine the Hilbert space:

$W_{2}^{4}([0,1] ; H)=\left\{u(t): u^{(4)}(t) \in L_{2}([0,1] ; H), \quad A^{4} u(t) \in L_{2}([0,1] ; H)\right\}$ with the scalar product

$$
(u, v)_{W_{2}^{4}([0,1] ; H)}=\int_{0}^{1}\left(u^{(4)}(t), v^{(4)}(t)\right)_{H} d t+\int_{0}^{1}\left(A^{4} u(t), A^{4} v(t)\right)_{H} d t
$$


and with the norm

$$
\|u\|_{W_{2}^{4}([0,1] ; H)}^{2}=\left\|u^{(4)}(t)\right\|_{L_{2}([0,1] ; H)}^{2}+\left\|A^{4} u(t)\right\|_{L_{2}([0,1] ; H)}^{2}
$$

From the theorem on traces [15], it follows that if $u(t) \in W_{2}^{4}([0,1] ; H)$ then $u^{(k)}(0) \in H_{4-k-\frac{1}{2}}, \quad u^{(k)}(1) \in H_{4-k-\frac{1}{2}}, \quad k=1,2,3$. Here and in the sequal, the derivative $u^{(k)} \equiv \frac{d^{k} u}{d t^{k}}$ is understood in the sense of distributions theory.

Let us consider the following boundary value problem:

$$
\begin{gathered}
L u=\frac{d^{4} u(t)}{d t^{4}}+A^{4} u(t)+\sum_{j=0}^{4} A_{4-j} u^{(j)}(t)=f(t), \quad t \in[0,1] \\
u(0)=u^{\prime}(0)=0, \quad u(1)=u^{\prime}(1)=0
\end{gathered}
$$

Here $f(t)$ and $u(t)$ are vector-functions determined almost everywhere in $[0,1]$ with the values in $H$.

We will assume that the coefficients of equation (2.1) satisfy the following conditions:

(1) $A$ is a self-adjoint, positive-definite operator in the space $H$.

(2) The operator $B_{j}=A_{j} A^{-j}, j=\overline{0,4}$ are bounded operators in the space $H$.

Definition 2.1. If the vector-function $u(t) \in W_{2}^{4}([0,1] ; H)$ satisfies the equation (2.1) almost everywhere in $[0,1]$ we call it a regular solution of equation (2.1).

Definition 2.2. If for any $f(t) \in L_{2}([0,1] ; H)$ there exists a regular solution of equation (2.1), for which boundary conditions (2.2) are fulfilled in the sense

$$
\begin{aligned}
& \lim _{t \rightarrow+0}\|u(t)\|_{H_{7 / 2}}=0, \lim _{t \rightarrow+0}\left\|u^{\prime}(t)\right\|_{H_{5 / 2}}=0, \\
& \lim _{t \rightarrow 1-0}\|u(t)\|_{H_{7 / 2}}=0, \lim _{t \rightarrow 1-0}\left\|u^{\prime}(t)\right\|_{H_{5 / 2}}=0,
\end{aligned}
$$

then the vector-function $u(t)$ is called a regular solution of boundary value problem (2.1), (2.2).

Definition 2.3. If for all $f(t) \in L_{2}([0,1] ; H)$ a boundary value problem has a regular solution and the estimation

$$
\|u\|_{W_{2}^{4}([0,1] ; H)} \leq c \cdot\|f\|_{L_{2}([0,1] ; H)}
$$

is fulfilled, then boundary value problem (2.1)-(2.2) is said to be uniquely solvable or regularity solvable.

In the space $\stackrel{\circ}{W}_{2}^{4}([0,1] ; H)$ we determine the following operators:

$$
\begin{aligned}
L_{0} u & =\frac{d^{4} u(t)}{d t^{4}}+A^{4} u(t), \quad u(t) \in \stackrel{\circ}{W}_{2}^{4}([0,1] ; H) \\
L_{1} u & =\sum_{j=0}^{4} A_{4-j} u^{(j)}(t), \quad u(t) \in \stackrel{\circ}{W}_{2}^{4}([0,1] ; H)
\end{aligned}
$$


In the papers $[19,20]$ it is shown that the operator $L_{0}$ realizes an isomorphism of the space $\stackrel{\circ}{W}_{2}^{4}([0,1] ; H)$ onto the space $L_{2}([0,1] ; H)$ and the operator $L_{0}^{-1}$ : $L_{2}([0,1] ; H) \rightarrow \stackrel{\circ}{W} \underset{2}{W}([0,1] ; H)$ is a bounded operator.

For proving the theorem on a regular solvability of problem (2.1)-(2.2) the following theorem on Kolmogorov type inequalities for intermediate derivatives of functions from the class $\stackrel{\circ}{W_{2}}([0,1] ; H)$ plays an important role.

\section{Main results}

The main result of this paper is the following theorem.

Theorem 3.1. Let the condition 1) be fulfilled and $A \geq 0$. Then for all $u(t) \in$ $\stackrel{\circ}{W}_{2}^{4}([0,1] ; H)$ we have the following inequalities

$$
\begin{aligned}
& \left\|A^{4} u\right\|_{L_{2}([0,1] ; H)} \leq c_{0} \cdot\left\|L_{0} u\right\|_{L_{2}([0,1] ; H)} \\
& \left\|A^{3} u^{\prime}\right\|_{L_{2}([0,1] ; H)} \leq c_{1} \cdot\left\|L_{0} u\right\|_{L_{2}([0,1] ; H)} \\
& \left\|A^{2} u^{\prime \prime}\right\|_{L_{2}([0,1] ; H)} \leq c_{2} \cdot\left\|L_{0} u\right\|_{L_{2}([0,1] ; H)} \\
& \left\|A u^{\prime \prime \prime}\right\|_{L_{2}([0,1] ; H)} \leq c_{3} \cdot\left\|L_{0} u\right\|_{L_{2}([0,1] ; H)} \\
& \left\|\frac{d^{4} u}{d t^{4}}\right\|_{L_{2}([0,1] ; H)} \leq c_{4} \cdot\left\|L_{0} u\right\|_{L_{2}([0,1] ; H)}
\end{aligned}
$$

where $c_{0}=c_{4}=1, \quad c_{2}=\frac{1}{2}, \quad c_{1}=\frac{1}{\sqrt{2}}, \quad c_{3}=\sqrt{3}+1+\frac{1}{2} \sqrt{10(2+\sqrt{3})}$.

Proof. For $u(t) \in \stackrel{\circ}{W}_{2}^{4}([0,1] ; H)$ we have the equality

$$
\begin{gathered}
\left\|L_{0} u\right\|_{L_{2}([0,1] ; H)}^{2}=\left\|\frac{d^{4} u}{d t^{4}}+A^{4} u\right\|_{L_{2}([0,1] ; H)}^{2}= \\
=\left\|\frac{d^{4} u}{d t^{4}}\right\|_{L_{2}([0,1] ; H)}^{2}+\left\|A^{4} u\right\|_{L_{2}([0,1] ; H)}^{2}+2 \operatorname{Re}\left(\frac{d^{4} u}{d t^{4}}, A^{4} u\right)_{L_{2}([0,1] ; H)} .
\end{gathered}
$$

On the other hand, integrating by parts we have

$$
\begin{gathered}
\left(\frac{d^{4} u}{d t^{4}}, A^{4} u\right)_{L_{2}([0,1] ; H)}=\int_{0}^{1}\left(\frac{d^{4} u(t)}{d t^{4}}, A^{4} u(t)\right) d t=\left.\left(A^{\frac{1}{2}} u^{\prime \prime \prime}(t), A^{\frac{7}{2}} u(t)\right)\right|_{0} ^{1}- \\
-\left.\left(A^{\frac{3}{2}} u^{\prime \prime}(t), A^{\frac{5}{2}} u^{\prime}(t)\right)\right|_{0} ^{1}+2 \int_{0}^{1}\left(A^{2} u^{\prime \prime}(t), A^{2} u^{\prime \prime}(t)\right) d t= \\
=\left.\left(A^{\frac{1}{2}} u^{\prime \prime}(t), A^{\frac{7}{2}} u(t)\right)\right|_{0} ^{1}-\left.\left(A^{\frac{2}{5}} u^{\prime \prime}(t), A^{\frac{2}{5}} u^{\prime}(t)\right)\right|_{0} ^{1}+2\left\|A^{2} u^{\prime \prime}(t)\right\|_{L_{2}([0,1] ; H)}^{2} .
\end{gathered}
$$

Since $u(t) \in \stackrel{\circ}{W_{2}}([0,1] ; H), u(0)=u^{\prime}(0)=u(1)=u^{\prime}(1)=0$, therefore

$$
\begin{gathered}
\left\|L_{0} u\right\|_{L_{2}([0,1] ; H)}^{2}= \\
=\left\|\frac{d^{4} u}{d t^{4}}\right\|_{L_{2}([0,1] ; H)}^{2}+\left\|A^{4} u\right\|_{L_{2}([0,1] ; H)}^{2}+2\left\|A^{2} \frac{d^{2} u}{d t^{2}}\right\|_{L_{2}([0,1] ; H)} .
\end{gathered}
$$


Hence we have

$$
\begin{aligned}
\left\|A^{4} u\right\|_{L_{2}([0,1] ; H)} & \leq\left\|L_{0} u\right\|_{L_{2}([0,1] ; H)}, \\
\left\|\frac{d^{4} u}{d t^{4}}\right\|_{L_{2}([0,1] ; H)} & \leq\left\|L_{0} u\right\|_{L_{2}([0,1] ; H)} .
\end{aligned}
$$

Thus inequalities (3.1) and (3.5) are proved.

Now we prove inequality (3.3).

It follows from inequality $(3.6)$ for $u(t) \in \stackrel{\circ}{W}_{2}^{4}([0,1] ; H)$ that

$$
\left\|L_{0} u\right\|_{L_{2}([0,1] ; H)}^{2}=\|u\|_{W_{2}^{4}([0,1] ; H)}^{2}+2\left\|A \frac{d^{2} u}{d t^{2}}\right\|_{L_{2}([0,1] ; H)} .
$$

Therefore,

$$
\|u\|_{W_{2}^{4}([0,1] ; H)}^{2}=\left\|L_{0} u\right\|_{L_{2}([0,1] ; H)}^{2}-2\left\|A \frac{d^{2} u}{d t^{2}}\right\|_{L_{2}([0,1] ; H)}^{2} .
$$

On the other hand we have

$$
\left\|A^{2} \frac{d^{2} u}{d t^{2}}\right\|_{L_{2}([0,1] ; H)}=\int_{0}^{1}\left(A^{2} \frac{d^{2} u(t)}{d t^{2}}, A^{2} \frac{d^{2} u(t)}{d t^{2}}\right) d t .
$$

Taking into account that $u(0)=u(1)=u^{\prime}(0)=u^{\prime}(1)=0$ after integration by parts we obtain

$$
\begin{gathered}
\left\|A^{2} \frac{d^{2} u}{d t^{2}}\right\|_{L_{2}([0,1] ; H)}^{2}=\left.\left(A^{\frac{5}{2}} u^{\prime}(t), A^{\frac{3}{2}} u^{\prime \prime}(t)\right)\right|_{0} ^{1}-\left.\left(A^{\frac{7}{2}} u(t), A^{\frac{1}{2}} u^{\prime \prime \prime}(t)\right)\right|_{0} ^{1}+ \\
+\int_{0}^{1}\left(A^{4} u(t), u^{(I V)}(t)\right) d t=\left[\left(A^{\frac{5}{2}} u^{\prime}(1), A^{\frac{3}{2}} u^{\prime \prime}(1)\right)-\left(A^{\frac{5}{2}} u^{\prime}(0), A^{\frac{3}{2}} u^{\prime \prime}(0)\right)\right]- \\
-\left[\left(A^{\frac{7}{2}} u(1), A^{\frac{1}{2}} u^{\prime \prime \prime}(1)\right)-\left(A^{\frac{7}{2}} u(0), A^{\frac{1}{2}} u^{\prime \prime \prime}(0)\right)\right]+ \\
+\int_{0}^{1}\left(A^{4} u(t), u^{(I V)}(t)\right) d t=\left(A^{4} u(t), \frac{d^{4} u}{d t^{4}}\right)_{L_{2}([0,1] ; H)} .
\end{gathered}
$$

Applying the Cauchy inequality we obtain

$$
\begin{gathered}
\left\|A \frac{d^{2} u}{d t^{2}}\right\|_{L_{2}([0,1] ; H)}^{2} \leq\left|\left(A^{4} u, \frac{d^{4} u}{d t^{4}}\right)_{L_{2}([0,1] ; H)}\right| \leq\left\|A^{4} u\right\|_{L_{2}([0,1] ; H)}\left\|\frac{d^{4} u}{d t^{4}}\right\|_{L_{2}([0,1] ; H)}< \\
<\frac{1}{2}\left(\left\|A^{4} u\right\|_{L_{2}([0,1] ; H)}^{2}+\left\|\frac{d^{4} u}{d t^{4}}\right\|_{L_{2}([0,1] ; H)}\right)=\frac{1}{2}\|u\|_{W_{2}([0,1] ; H)}^{2}
\end{gathered}
$$

Then from equality (3.7) we obtain

$$
\frac{1}{2}\|u\|_{W_{2}^{4}([0,1] ; H)}^{2}=\frac{1}{2}\left\|L_{0} u\right\|_{L_{2}([0,1] ; H)}^{2}-\left\|A \frac{d^{2} u}{d t^{2}}\right\| \geq\left\|A^{2} \frac{d^{2} u}{d t^{2}}\right\|_{L_{2}([0,1] ; H)}
$$

Hence

$$
\left\|A^{2} \frac{d^{2} u}{d t^{2}}\right\|_{L_{2}([0,1] ; H)}^{2} \leq \frac{1}{2}\left\|L_{0} u\right\|_{L_{2}([0,1] ; H)}^{2}
$$


i.e.

Consequently,

$$
\left\|A^{2} \frac{d^{2} u}{d t^{2}}\right\|_{L_{2}([0,1] ; H)}^{2} \leq \frac{1}{4}\left\|L_{0} u\right\|_{L_{2}([0,1] ; H)}^{2}
$$

$$
\left\|A^{2} \frac{d^{2} u}{d t^{2}}\right\|_{L_{2}([0,1] ; H)} \leq \frac{1}{2}\left\|L_{0} u\right\|_{L_{2}([0,1] ; H)} .
$$

Thus, inequality (3.3) is proved.

Now we prove inequality (3.2).

Let $u(t) \in \stackrel{\circ}{W_{2}^{4}}([0,1] ; H)$. Applying integration by parts and having in view the condition, $u(0)=u(1)=u^{\prime}(0)=u^{\prime}(1)=0$ we get

$$
\begin{gathered}
\left\|A^{3} \frac{d u}{d t}\right\|_{L_{2}([0,1] ; H)}^{2}=\int_{0}^{1}\left(A^{3} \frac{d u}{d t}, A^{3} \frac{d u}{d t}\right)_{H} d t=\left.\left(A^{\frac{7}{2}} u(t), A^{\frac{5}{2}} u^{\prime}(t)\right)\right|_{0} ^{1}- \\
-\int_{0}^{1}\left(A^{4} u(t), A^{2} \frac{d^{2} u}{d t^{2}}\right)_{H} d t=\left(A^{\frac{7}{2}} u(1), A^{\frac{5}{2}} u(1)\right)-\left(A^{\frac{7}{2}} u(0), A^{\frac{5}{2}} u(0)\right)- \\
-\int_{0}^{1}\left(A^{4} u(t), A^{2} \frac{d^{2} u}{d t^{2}}\right)_{H} d t=-\left(A^{4} u, A^{2} \frac{d^{2} u}{d t^{2}}\right)_{L_{2}([0,1] ; H)} .
\end{gathered}
$$

Hence we have

$$
\left\|A^{3} \frac{d u}{d t}\right\|_{L_{2}([0,1] ; H)} \leq\left\|A^{4} u\right\|_{L_{2}([0,1] ; H)} \cdot\left\|A^{2} \frac{d^{2} u}{d t^{2}}\right\|_{L_{2}([0,1] ; H)} .
$$

If we use inequalities (3.1), (3.3), we obtain that

$\left\|A^{3} \frac{d u}{d t}\right\|_{L_{2}([0,1] ; H)}^{2} \leq\left\|L_{0 u}\right\|_{L_{2}([0,1] ; H)} \cdot \frac{1}{2}\left\|L_{0} u\right\|_{L_{2}([0,1] ; H)} \leq \frac{1}{2}\left\|L_{0} u\right\|_{L_{2}([0,1] ; H)}^{2}$.

Hence

$$
\left\|A^{3} \frac{d u}{d t}\right\|_{L_{2}([0,1] ; H)} \leq \frac{1}{\sqrt{2}}\left\|L_{0} u\right\|_{L_{2}([0,1] ; H)} .
$$

Thus, inequality (3.2) is proved.

Now we prove inequality (3.4).

For $u(t) \in \stackrel{\circ}{W}_{2}^{4}([0,1] ; H)$ we have

$$
\begin{gathered}
\left\|A \frac{d^{3} u(t)}{d t^{3}}\right\|_{L_{2}([0,1] ; H)}^{2}=\int_{0}^{1}\left(A \frac{d^{3} u(t)}{d t^{3}}, A \frac{d^{3} u(t)}{d t^{3}}\right)_{H} d t=\left.\left(A^{\frac{5}{2}} u^{\prime \prime}(t), A^{\frac{1}{2}} u^{\prime \prime \prime}(t)\right)\right|_{0} ^{1}- \\
-\int_{0}^{1}\left(A^{2} \frac{d^{2} u(t)}{d t^{2}}, \frac{d^{4} u(t)}{d t^{4}}\right)_{H} d t=\left(A^{\frac{5}{2}} u^{\prime \prime}(1), A^{\frac{1}{2}} u^{\prime \prime \prime}(1)\right)- \\
-\left(A^{\frac{5}{2}} u^{\prime \prime}(0), A^{\frac{1}{2}} u^{\prime \prime \prime}(0)\right)-\left(A^{2} \frac{d^{2} u}{d t^{2}}, \frac{d^{4} u}{d t^{4}}\right)_{L_{2}([0,1] ; H)} .
\end{gathered}
$$

Hence we have

$$
\left\|A \frac{d^{3} u}{d t^{3}}\right\|_{L_{2}([0,1] ; H)}^{2} \leq\left\|A^{\frac{5}{2}} u^{\prime \prime}(1)\right\| \cdot\left\|A^{\frac{1}{2}} u^{\prime \prime \prime}(1)\right\|+
$$


ON KOLMOGOROV TYPE INEQUALITIES FOR THE NORMS OF OPERATORS . . 149

$$
+\left\|A^{\frac{5}{2}} u^{\prime \prime}(0)\right\| \cdot\left\|A^{\frac{1}{2}} u^{\prime \prime \prime}(0)\right\|+\left\|A^{2} \frac{d^{2} u}{d t^{2}}\right\|_{L_{2}([0,1] ; H)} \cdot\left\|\frac{d^{4} u}{d t^{4}}\right\|_{L_{2}([0,1] ; H)} .
$$

Applying the Cauchy inequality, we obtain that

$$
\begin{gathered}
\left\|A \frac{d^{3} u}{d t^{3}}\right\|_{L_{2}([0,1] ; H)}^{2} \leq \frac{1}{2}\left(\left\|A^{\frac{5}{2}} u^{\prime \prime}(1)\right\|^{2}+\left\|A^{\frac{1}{2}} u^{\prime \prime \prime}(1)\right\|^{2}\right)+ \\
+\frac{1}{2}\left(\left\|A^{\frac{5}{2}} u^{\prime \prime}(0)\right\|^{2}+\left\|A^{\frac{1}{2}} u^{\prime \prime \prime}(0)\right\|^{2}\right)+\frac{1}{2}\left\|A^{2} \frac{d^{2} u}{d t^{2}}\right\|_{L_{2}([0,1] ; H)}+ \\
+\frac{1}{2}\left\|\frac{d^{4} u}{d t^{4}}\right\|_{L_{2}([0,1] ; H)}^{2} \leq \frac{1}{2}\left(\left\|A^{\frac{5}{2}} u^{\prime \prime}(1)\right\|^{2}+\left\|A^{\frac{1}{2}} u^{\prime \prime \prime}(1)\right\|^{2}\right)+ \\
+\frac{1}{2}\left(\left\|A^{\frac{5}{2}} u^{\prime \prime}(0)\right\|^{2}+\left\|A^{\frac{1}{2}} u^{\prime \prime \prime}(0)\right\|^{2}\right)+\frac{1}{2}\|u\|_{W_{2}^{4}([0,1] ; H)} .
\end{gathered}
$$

From inequality (3.7) it follows that

$$
\|u\|_{W_{2}^{4}([0,1] ; H)} \leq\left\|L_{0} u\right\|_{L_{2}([0,1] ; H)} .
$$

Therefore for $\tau>0$ we have

$$
\begin{aligned}
& \left\|A \frac{d^{3} u}{d t^{3}}\right\|_{L_{2}([0,1] ; H)}^{2} \leq \frac{\tau}{2}\left(\left\|A^{\frac{5}{2}} u^{\prime \prime}(1)\right\|^{2}+\frac{1}{2 \tau}\left\|A^{\frac{1}{2}} u^{\prime \prime \prime}(1)\right\|^{2}\right)+ \\
& +\frac{\tau}{2}\left(\left\|A^{\frac{5}{2}} u^{\prime \prime}(0)\right\|^{2}+\frac{1}{2 \tau}\left\|A^{\frac{1}{2}} u^{\prime \prime \prime}(0)\right\|^{2}\right)+\frac{1}{2}\left\|L_{0} u\right\|_{L_{2}([0,1] ; H)}^{2} .
\end{aligned}
$$

Note that if $u(t) \in \stackrel{\circ}{W_{2}}([0,1] ; H)$, then $\frac{d^{3} u}{d t^{3}} \in L_{2}([0,1] ; H)$.

Thus $t u^{\prime \prime \prime}(t) \in L_{2}([0,1] ; H), \quad t \in[0,1]$.

Then we can easily see that

$$
\left\|A^{\frac{1}{2}} u^{\prime \prime \prime}(t)\right\|^{2}=2 \operatorname{Re} \int_{0}^{1}\left(\frac{d^{4} u(t)}{d t^{4}}, t A \frac{d^{3} u(t)}{d t^{3}}\right) d t+\left\|A^{\frac{1}{2}} u^{\prime \prime \prime}(t)\right\|_{L_{2}([0,1] ; H)}^{2}
$$

Indeed,

or

$$
\begin{gathered}
\int_{0}^{1}\left(\frac{d^{4} u}{d t^{4}}, t A \frac{d^{3} u(t)}{d t^{3}}\right) d t=\left.\left(A^{\frac{1}{2}} u^{\prime \prime \prime}(t), t A^{\frac{1}{2}} u^{\prime \prime \prime}(t)\right)\right|_{0} ^{1}- \\
-\int_{0}^{1}\left(A^{\frac{1}{2}} \frac{d^{3} u}{d t^{3}}, A^{\frac{1}{2}} \frac{d^{3} u}{d t^{3}}\right)_{H} d t-\int_{0}^{1}\left(A \frac{d^{3} u}{d t^{3}}, t \frac{d^{4} u}{d t^{4}}\right) d t= \\
=\left(A^{\frac{1}{2}} u^{\prime \prime \prime}(1), A^{\frac{1}{2}} u^{\prime \prime \prime}(1)\right)-\left(A^{\frac{1}{2}} u^{\prime \prime \prime}(0), 0 \cdot A^{\frac{1}{2}} u^{\prime \prime \prime}(0)\right)- \\
-\int_{0}^{1}\left(A \frac{d^{3} u}{d t^{3}}, A \frac{d^{3} u}{d t^{3}}\right)_{H} d t-\int_{0}^{1}\left(t A \frac{d^{3} u}{d t^{3}}, \frac{d^{4} u}{d t^{4}}\right) d t
\end{gathered}
$$

$\left(\frac{d^{4} u}{d t^{4}}, t A \frac{d^{3} u(t)}{d t^{3}}\right)_{L_{2}([0,1] ; H)}=\left\|A^{\frac{1}{2}} u^{\prime \prime \prime}(1)\right\|^{2}-\left(t A \frac{d^{3} u(t)}{d t^{3}}, \frac{d^{4} u}{d t^{4}}\right)_{L_{2}([0,1] ; H)}$.

Hence we have

$$
\left\|A^{\frac{1}{2}} u^{\prime \prime \prime}(1)\right\|^{2}=2 R e\left(\frac{d^{4} u}{d t^{4}}, t A \frac{d^{3} u(t)}{d t^{3}}\right)_{L_{2}([0,1] ; H)}+\left\|A^{\frac{1}{2}} u^{\prime \prime \prime}(1)\right\|_{L_{2}([0,1] ; H)}^{2} .
$$


Now we show that

$$
\begin{gathered}
\left\|A^{\frac{1}{2}} u^{\prime \prime \prime}(0)\right\|^{2}= \\
=2 \operatorname{Re} \int_{0}^{1}\left(\frac{d^{4} u}{d t^{4}},(1-t) A \frac{d^{3} u(t)}{d t^{3}}\right)_{H} d t+\left\|A^{\frac{1}{2}} \frac{d^{3} u}{d t^{3}}\right\|_{L_{2}([0,1] ; H)}^{2} .
\end{gathered}
$$

Note that if $u(t) \in \stackrel{\circ}{W_{2}^{4}}([0,1] ; H)$, then for $0<t<1,(1-t) A \frac{d^{3} u}{d t^{3}} \in L_{2}([0,1] ; H)$.

By means of integration by parts, we have

$$
\begin{aligned}
& \int_{0}^{1}(\left.\frac{d^{4} u}{d t^{4}},(1-t) A \frac{d^{3} u}{d t^{3}}\right)_{H} d t=\left.\left(A^{\frac{1}{2}} u^{\prime \prime \prime}(t),(1-t) A^{\frac{1}{2}} u^{\prime \prime \prime}(t)\right)\right|_{0} ^{1}- \\
&-\int_{0}^{1}\left(\frac{d^{3} u}{d t^{3}}, A \frac{d^{3} u}{d t^{3}}\right) d t-\int_{0}^{1}\left(A \frac{d^{3} u}{d t^{3}},(1-t) \frac{d^{4} u}{d t^{4}}\right) d t= \\
&=\left(A^{\frac{1}{2}} u^{\prime \prime \prime}(1), 0 \cdot A^{\frac{1}{2}} u^{\prime \prime \prime}(1)\right)-\left(A^{\frac{1}{2}} u^{\prime \prime \prime}(0), A^{\frac{1}{2}} u^{\prime \prime \prime}(0)\right)+ \\
&+\int_{0}^{1}\left(\frac{d^{3} u}{d t^{3}}, A \frac{d^{3} u}{d t^{3}}\right)_{H} d t-\int_{0}^{1}\left((1-t) A \frac{d^{3} u}{d t^{3}}, \frac{d^{4} u}{d t^{4}}\right) d t= \\
&=-\left\|A^{\frac{1}{2}} u^{\prime \prime \prime}(0)\right\|^{2}-\int_{0}^{1}\left((1-t) A \frac{d^{3} u}{d t^{3}}, \frac{d^{4} u}{d t^{4}}\right)_{H} d t+\left\|A^{\frac{1}{2}} \frac{d^{3} u}{d t^{3}}\right\|_{L_{2}([0,1] ; H)}^{2}
\end{aligned}
$$

Hence we have

$$
\int_{0}^{1}\left(\frac{d^{4} u}{d t^{4}},(1-t) A \frac{d^{3} u}{d t^{3}}\right)_{H} d t+\int_{0}^{1}\left((1-t) A \frac{d^{3} u}{d t^{3}}, \frac{d^{4} u}{d t^{4}}\right) d t=-\left\|A^{\frac{1}{2}} u^{\prime \prime \prime}(0)\right\|^{2}
$$

i.e.

$$
\left\|A^{\frac{1}{2}} u^{\prime \prime \prime}(0)\right\|^{2}=-2 \operatorname{Re} \int_{0}^{1}\left(\frac{d^{4} u}{d t^{4}},(1-t) A \frac{d^{3} u}{d t^{3}}\right) d t+\left\|A^{\frac{1}{2}} \frac{d^{3} u}{d t^{3}}\right\|_{L_{2}([0,1] ; H)} .
$$

Thus we obtain that equality (3.10) is valid.

Now we prove the following equalities

$$
\left\|A^{\frac{3}{2}} u^{\prime \prime}(1)\right\|^{2}=2 R e \int_{0}^{1}\left(A \frac{d^{3} u}{d t^{3}}, t A^{2} \frac{d^{2} u}{d t^{2}}\right) d t+\left\|A^{\frac{3}{2}} \frac{d^{2} u}{d t^{2}}\right\|_{L_{2}([0,1] ; H)}
$$

and

$$
\left\|A^{\frac{3}{2}} u^{\prime \prime}(0)\right\|^{2}=2 \operatorname{Re} \int_{0}^{1}\left(A \frac{d^{3} u}{d t^{3}},(1-t) A \frac{d^{2} u}{d t^{2}}\right) d t+\left\|A^{\frac{3}{2}} \frac{d^{2} u}{d t^{2}}\right\|_{L_{2}([0,1] ; H)} .
$$

Let $u(t) \in \stackrel{\circ}{W}_{2}^{4}([0,1] ; H)$. Then we have

$$
\begin{gathered}
\int_{0}^{1}\left(A \frac{d^{3} u}{d t^{3}}, t A^{2} \frac{d^{2} u}{d t^{2}}\right) d t=\left.\left(A \frac{d^{2} u}{d t^{2}}, t A^{2} \frac{d^{2} u}{d t^{2}}\right)\right|_{0} ^{1}- \\
-\int_{0}^{1}\left(A \frac{d^{2} u}{d t^{2}}, A^{2} \frac{d^{2} u}{d t^{2}}\right) d t-\int_{0}^{1}\left(t A^{2} \frac{d^{2} u}{d t^{2}}, A \frac{d^{3} u}{d t^{3}}\right) d t= \\
=\left(A u^{\prime \prime}(1), t A^{2} u^{\prime \prime}(1)\right)-\left(A u^{\prime \prime}(0), 0 \cdot A^{2} u^{\prime \prime}(0)\right)- \\
-\int_{0}^{1}\left(A^{\frac{3}{2}} \frac{d^{2} u}{d t^{2}}, A^{\frac{3}{2}} \frac{d^{2} u}{d t^{2}}\right) d t-\int_{0}^{1}\left(t A^{2} \frac{d^{2} u}{d t^{2}}, A \frac{d^{3} u}{d t^{3}}\right) d t .
\end{gathered}
$$


Hence we have

or

$$
\begin{gathered}
\int_{0}^{1}\left(A \frac{d^{3} u}{d t^{3}}, t A^{2} \frac{d^{2} u}{d t^{2}}\right) d t=\int_{0}^{1}\left(t A^{2} \frac{d^{2} u}{d t^{2}}, A \frac{d^{3} u}{d t^{3}}\right) d t+ \\
+\left\|A^{\frac{3}{2}} u^{\prime \prime}(1)\right\|^{2}-\int_{0}^{1}\left(A^{\frac{3}{2}} \frac{d^{2} u}{d t^{2}}, A^{\frac{3}{2}} \frac{d^{2} u}{d t^{2}}\right) d t
\end{gathered}
$$

$$
\left\|A^{\frac{3}{2}} u^{\prime \prime}(1)\right\|^{2}=2 \operatorname{Re} \int_{0}^{1}\left(A \frac{d^{3} u}{d t^{3}}, t A^{2} \frac{d^{2} u}{d t^{2}}\right) d t+\left\|A^{\frac{3}{2}} \frac{d^{2} u}{d t^{2}}\right\|_{L_{2}([0,1] ; H)} .
$$

Equality (3.11) is proved.

Let us prove equality $(3.12)$. Let $u(t) \in \stackrel{\circ}{W}_{2}^{4}([0,1] ; H)$

$$
\begin{gathered}
\int_{0}^{1}\left(A \frac{d^{3} u}{d t^{3}},(1-t) A^{2} \frac{d^{2} u}{d t^{2}}\right) d t=\left.\left(A^{\frac{3}{2}} u^{\prime \prime}(t),(1-t) A^{\frac{3}{2}} u^{\prime \prime}(t)\right)\right|_{0} ^{1}- \\
-\int_{0}^{1}\left(A \frac{d^{2} u}{d t^{2}}, A^{2} \frac{d^{2} u}{d t^{2}}\right) d t=-\int_{0}^{1}(1-t)\left(A^{2} \frac{d^{2} u}{d t^{2}}, A \frac{d^{3} u}{d t^{3}}\right) d t .
\end{gathered}
$$

Hence we have

$$
\begin{gathered}
\int_{0}^{1}\left(A \frac{d^{3} u}{d t^{3}},(1-t) A^{2} \frac{d^{2} u}{d t^{2}}\right) d t+\int_{0}^{1}\left((1-t) A^{2} \frac{d^{2} u}{d t^{2}}, A \frac{d^{3} u}{d t^{3}}\right) d t= \\
=\left(A^{\frac{3}{2}} u^{\prime \prime}(1), 0 \cdot A^{\frac{3}{2}} u^{\prime \prime}(1)\right)-\left(A^{\frac{3}{2}} u^{\prime \prime}(0), A^{\frac{3}{2}} u^{\prime \prime}(0)\right)+\int_{0}^{1}\left(A^{\frac{3}{2}} \frac{d^{2} u}{d t^{2}}, A^{\frac{3}{2}} \frac{d^{2} u}{d t^{2}}\right) d t .
\end{gathered}
$$

or

$$
\left\|A^{\frac{3}{2}} u^{\prime \prime}(0)\right\|^{2}=2 \operatorname{Re} \int_{0}^{1}\left(A \frac{d^{3} u}{d t^{3}},(1-t) A^{2} \frac{d^{2} u}{d t^{2}}\right) d t+\left\|A^{\frac{3}{2}} \frac{d^{2} u}{d t^{2}}\right\|_{L_{2}([0,1] ; H)} .
$$

Equality (3.12) is proved.

From equality (3.9) we obtain that

$$
\begin{aligned}
& \left\|A^{\frac{1}{2}} u^{\prime \prime \prime}(1)\right\|^{2} \leq 2\left\|\frac{d^{4} u}{d t^{4}}\right\|_{L_{2}([0,1] ; H)} \cdot\left\|t A \frac{d^{3} u}{d t^{3}}\right\|_{L_{2}([0,1] ; H)}+\left\|A^{-\frac{1}{2}} A \frac{d^{3} u}{d t^{3}}\right\|_{L_{2}([0,1] ; H)} \leq \\
& \leq 2\left\|\frac{d^{4} u}{d t^{4}}\right\|_{L_{2}([0,1] ; H)} \cdot\left\|A \frac{d^{3} u}{d t^{3}}\right\|_{L_{2}([0,1] ; H)}+\left\|A^{-\frac{1}{2}}\right\|^{2} \cdot\left\|A \frac{d^{3} u}{d t^{3}}\right\|_{L_{2}([0,1] ; H)} \cdot(3.13)
\end{aligned}
$$

Since $A \geq E$, then $\left\|A^{-\frac{1}{2}}\right\|^{2} \leq 1$. Therefore,

$$
\left\|A^{\frac{1}{2}} u^{\prime \prime \prime}(1)\right\|^{2} \leq 2\left\|\frac{d^{4} u}{d t^{4}}\right\|_{L_{2}([0,1] ; H)} \cdot\left\|A \frac{d^{3} u}{d t^{3}}\right\|_{L_{2}([0,1] ; H)}+\left\|A \frac{d^{3} u}{d t^{3}}\right\|_{L_{2}([0,1] ; H)}^{2} .
$$

From equality (3.10) we have

$$
\begin{gathered}
\left\|A^{\frac{1}{2}} u^{\prime \prime \prime}(0)\right\|^{2} \leq 2\left\|\frac{d^{4} u}{d t^{4}}\right\|_{L_{2}([0,1] ; H)} \cdot\left\|(1-t) A \frac{d^{3} u}{d t^{3}}\right\|_{L_{2}([0,1] ; H)}+ \\
+\left\|A^{-\frac{1}{2}}\right\| \cdot\left\|A \frac{d^{3} u}{d t^{3}}\right\|_{L_{2}([0,1] ; H)} \leq 2\left\|\frac{d^{4} u}{d t^{4}}\right\|_{L_{2}([0,1] ; H)} \times \\
\times\left\|A \frac{d^{3} u}{d t^{3}}\right\|_{L_{2}([0,1] ; H)}+\left\|A \frac{d^{3} u}{d t^{3}}\right\|_{L_{2}([0,1] ; H)}^{2} .
\end{gathered}
$$


From equality (3.11) it follows that

$$
\begin{gathered}
\left\|A^{\frac{3}{2}} u^{\prime \prime}(1)\right\|^{2} \leq 2\left\|A \frac{d^{3} u}{d t^{3}}\right\|_{L_{2}([0,1] ; H)}^{2} \cdot\left\|A^{2} \frac{d^{2} u}{d t^{2}}\right\|_{L_{2}([0,1] ; H)}+\left\|A^{-\frac{1}{2}} \cdot A \frac{d^{2} u}{d t^{2}}\right\|_{L_{2}([0,1] ; H)}^{2} \leq \\
\quad \leq 2\left\|A \frac{d^{3} u}{d t^{4}}\right\|_{L_{2}([0,1] ; H)} \cdot\left\|A^{2} \frac{d^{2} u}{d t^{2}}\right\|_{L_{2}([0,1] ; H)}+\left\|A^{2} \frac{d^{2} u}{d t^{2}}\right\|_{L_{2}([0,1] ; H)}^{2} \cdot
\end{gathered}
$$

In the similar way we get

$$
\begin{gathered}
\left\|A^{\frac{3}{2}} u^{\prime \prime}(0)\right\|^{2} \leq 2\left\|A \frac{d^{3} u}{d t^{3}}\right\|_{L_{2}([0,1] ; H)} \cdot\left\|A^{2} \frac{d^{2} u}{d t^{2}}\right\|_{L_{2}([0,1] ; H)}+\left\|A^{-\frac{1}{2}} A^{2} \frac{d^{2} u}{d t^{2}}\right\|_{L_{2}([0,1] ; H)} \leq \\
\leq 2\left\|A \frac{d^{3} u}{d t^{3}}\right\|_{L_{2}([0,1] ; H)} \cdot\left\|A^{2} \frac{d^{2} u}{d t^{2}}\right\|_{L_{2}([0,1] ; H)}+\left\|A^{2} \frac{d^{2} u}{d t^{2}}\right\|_{L_{2}([0,1] ; H)} \cdot
\end{gathered}
$$

Taking into account inequalities (3.13)-(3.16) in inequality (3.8) for $\tau>0$ we get

$$
\begin{aligned}
& \leq \frac{\tau}{2}\left(2\left\|\frac{d^{4} u}{d t^{4}}\right\|_{L_{2}([0,1] ; H)} \cdot\left\|A \frac{d^{3} u}{d t^{3}}\right\|_{L_{2}([0,1] ; H)}^{2} \|_{t^{3}} \leq\right. \\
& +\frac{\tau}{2}\left(2\left\|\frac{d^{4} u}{d t^{4}}\right\|_{L_{2}([0,1] ; H)} \cdot\left\|A \frac{d^{3} u}{d t^{3}}\right\|_{L_{2}([0,1] ; H)}+\left\|A \frac{d^{3} u}{d t^{3}}\right\|_{L_{2}([0,1] ; H)}\right)+ \\
& +\frac{1}{2 \tau}\left(2\left\|A \frac{d^{3} u}{d t^{3}}\right\|_{L_{2}([0,1] ; H)}\right)+ \\
& +\frac{1}{2 \tau}\left(2\left\|A \frac{d^{3} u}{d t^{3}}\right\|_{L_{2}([0,1] ; H)} \cdot\left\|A^{2} \frac{d^{2} u}{d t^{2}}\right\|_{L_{2}([0,1] ; H)}+\left\|A \frac{d^{2} u}{d t^{2}}\right\|_{L_{2}([0,1] ; H)}\right)+ \\
& \left.=2 \tau\left\|\frac{d^{3} u}{d t^{4}}\right\|_{L_{2}([0,1] ; H)} \cdot\left\|A \frac{d^{3} u}{d t^{3}}\right\|_{L_{2}([0,1] ; H)}+\left\|A \frac{d^{2} u}{d t^{2}}\right\|_{L_{2}([0,1] ; H)}+\left\|A \frac{d^{2} u}{d t^{2}}\right\|_{L_{2}([0,1] ; H)}\right)= \\
& +\frac{2}{\tau}\left\|A \frac{d^{3} u}{d t^{3}}\right\|_{L_{2}([0,1] ; H)} \cdot\left\|A^{2} \frac{d^{2} u}{d t^{2}}\right\|_{L_{2}([0,1] ; H)}+ \\
& +A_{L_{2}([0,1] ; H)}+\frac{1}{\tau}\left\|A^{2} \frac{d^{2} u}{d t^{2}}\right\|_{L_{2}([0,1] ; H)} \cdot
\end{aligned}
$$

Taking into account

and

$$
\left\|\frac{d^{4} u}{d t^{4}}\right\|_{L_{2}([0,1] ; H)} \leq\left\|L_{0} u\right\|_{L_{2}([0,1] ; H)}
$$

$$
\left\|A^{2} \frac{d^{2} u}{d t^{2}}\right\|_{L_{2}([0,1] ; H)} \leq \frac{1}{2}\left\|L_{0} u\right\|_{L_{2}([0,1] ; H)}
$$

from inequality (3.17) we get

$$
\begin{gathered}
\left\|A \frac{d^{3} u}{d t^{3}}\right\|_{L_{2}([0,1] ; H)}^{2} \leq 2 \tau\left\|A \frac{d^{3} u}{d t^{3}}\right\|_{L_{2}([0,1] ; H)} \cdot\left\|L_{0} u\right\|_{L_{2}([0,1] ; H)}+\tau\left\|A \frac{d^{3} u}{d t^{3}}\right\|_{L_{2}([0,1] ; H)}+ \\
+\frac{1}{\tau}\left\|A \frac{d^{3} u}{d t^{3}}\right\|_{L_{2}([0,1] ; H)} \cdot\left\|L_{0} u\right\|_{L_{2}([0,1] ; H)}+\frac{1}{4 \tau}\left\|L_{0} u\right\|_{L_{2}([0,1] ; H)}^{2}+
\end{gathered}
$$




$$
+\frac{1}{2}\left\|L_{0} u\right\|_{L_{2}([0,1] ; H)}^{2}+\frac{1}{2}\left\|L_{0} u\right\|_{L_{2}([0,1] ; H)}^{2} .
$$

Hence, for $\tau<1$ we have

$$
\begin{gathered}
(1-\tau)\left\|A \frac{d^{3} u}{d t^{3}}\right\|_{L_{2}([0,1] ; H)} \leq\left(2 \tau+\frac{1}{\tau}\right)\left\|L_{0} u\right\|_{L_{2}([0,1] ; H)}+ \\
+\left(\frac{1}{4 \tau}+\frac{1}{2}\right)\left\|L_{0} u\right\|_{L_{2}([0,1] ; H)}^{2} .
\end{gathered}
$$

For $\tau<1\left\|A \frac{d^{3} u}{d t^{3}}\right\|$ satisfies the inequality

$$
\begin{aligned}
& \left\|A \frac{d^{3} u}{d t^{3}}\right\|_{L_{2}([0,1] ; H)} \leq \frac{2 \tau+\frac{1}{\tau}}{1-\tau}\left\|L_{0} u\right\|_{L_{2}([0,1] ; H)} \times \\
& \times\left\|A \frac{d^{3} u}{d t^{3}}\right\|_{L_{2}([0,1] ; H)}+\frac{\frac{1}{4 \tau}+\frac{1}{2}}{1-\tau}\left\|L_{0} u\right\|_{L_{2}([0,1] ; H)}^{2} .
\end{aligned}
$$

Denote by $\varphi(\tau)$ the function

$$
\varphi(\tau)=\frac{2 \tau+\frac{1}{\tau}}{1-\tau}=\frac{2 \tau^{2}+1}{\tau(1-\tau)}, \quad \tau \in(0,1)
$$

and find minimal value $\varphi(\tau)$.

$$
\varphi^{\prime}(\tau)=\frac{4 \tau\left(\tau-\tau^{2}\right)-\left(2 \tau^{2}+1\right)(1-2 \tau)}{\left(\tau-\tau^{2}\right)^{2}}=\frac{2 \tau^{2}+2 \tau-1}{\left(\tau-\tau^{2}\right)^{2}} .
$$

From the equation $2 \tau^{2}+2 \tau-1=0$ we find $\tau=\frac{-1+\sqrt{3}}{2}$. At $\tau=\frac{-1+\sqrt{3}}{2}$ for $\left\|A \frac{d^{3} u}{d t^{3}}\right\|_{L_{2}([0,1] ; H)}$ we get the inequality

$$
\begin{gathered}
\left\|A \frac{d^{3} u}{d t^{3}}\right\|_{L_{2}([0,1] ; H)} \leq \frac{2 \cdot\left(\frac{\sqrt{3}-1}{2}\right)^{2}+1}{\frac{\sqrt{3}-1}{2}-\left(\frac{\sqrt{3}-1}{2}\right)^{2}} \cdot\left\|A \frac{d^{3} u}{d t^{3}}\right\|_{L_{2}([0,1] ; H)}^{2} \times \\
\times\left\|L_{0} u\right\|_{L_{2}([0,1] ; H)}^{2}++\left(\left(\frac{1}{2}+\frac{1}{4} \cdot \frac{2}{\sqrt{3}-1}\right) \cdot \frac{1}{1-\frac{\sqrt{3}-1}{2}}\right) \cdot\left\|L_{0} u\right\|_{L_{2}([0,1] ; H)}^{2}
\end{gathered}
$$

or

$$
\begin{gathered}
\left\|A \frac{d^{3} u}{d t^{3}}\right\|_{L_{2}([0,1] ; H)} \leq \frac{2 \cdot \frac{4-2 \sqrt{3}}{4}+1}{\frac{\sqrt{3}-1}{2}-\frac{4-2 \sqrt{3}}{4}} \cdot\left\|A \frac{d^{3} u}{d t^{3}}\right\|_{L_{2}([0,1] ; H)}^{2} \cdot\left\|L_{0} u\right\|_{L_{2}([0,1] ; H)}^{2}+ \\
+\left(\frac{1}{2}+\frac{\sqrt{3}+1}{4}\right) \cdot \frac{2}{3-\sqrt{3}} \cdot\left\|L_{0} u\right\|_{L_{2}([0,1] ; H)}^{2} \cdot
\end{gathered}
$$

Hence we have

$$
\begin{aligned}
\left\|A \frac{d^{3} u}{d t^{3}}\right\|_{L_{2}([0,1] ; H)}^{2} \leq & 2(\sqrt{3}+1) \cdot\left\|A \frac{d^{3} u}{d t^{3}}\right\|_{L_{2}([0,1] ; H)} \cdot\left\|L_{0} u\right\|_{L_{2}([0,1] ; H)}+ \\
& +\frac{2+\sqrt{3}}{2} \cdot\left\|L_{0} u\right\|_{L_{2}([0,1] ; H)}^{2} \cdot
\end{aligned}
$$


We can rewrite this inequality in the following form

$$
\begin{aligned}
& \left(\left\|A \frac{d^{3} u}{d t^{3}}\right\|_{L_{2}([0,1] ; H)}^{2}-(\sqrt{3}+1) \cdot\left\|L_{0} u\right\|_{L_{2}([0,1] ; H)}\right)^{2} \leq \\
\leq & (\sqrt{3}+1) \cdot\left\|L_{0} u\right\|_{L_{2}([0,1] ; H)}^{2}+\frac{2+\sqrt{3}}{2} \cdot\left\|L_{0} u\right\|_{L_{2}([0,1] ; H)}^{2} .
\end{aligned}
$$

Hence we finally have

$$
\left\|A \frac{d^{3} u}{d t^{3}}\right\|_{L_{2}([0,1] ; H)}^{2} \leq\left(\sqrt{3}+1+\frac{1}{2} \sqrt{10(2+\sqrt{3})}\right) \cdot\left\|L_{0} u\right\|_{L_{2}([0,1] ; H)} .
$$

Thus, the theorem is completely proved.

Remark 3.1. It is easy to see that $c_{3}=\sqrt{3}+1+\frac{1}{2} \sqrt{10(2+\sqrt{3})}<6$.

Remark 3.2. As can be seen from the proof of the theorem, when obtaining inequalities (3.1), (3.2), (3.3) and (3.5) the condition $A \geq E$ is not used, but when proving inequality (3.4) this condition is used. Thus, the values $c_{0}, c_{1}, c_{2}, c_{4}$ are universal i.e. they are independent of the operator $A$. Only $c_{3}$ is not universal and depends on the operator $A$.

\section{Acknowledgements}

The authors express their gratitude to professor M. Bayramoglu and professor S.S. Mirzoyev for useful discussions of the obtained results.

\section{References}

[1] M.S.Agranovich, M.I.Vishik, Elliptic problems with a parameter and general parabolic problems, UMN 19 (1964) no. 3, 53-165.

[2] A.R.Aliyev, Boundary value problems for a class of operator-differential equations with variable coefficients, Matem. zametki 74 (2003), issue 6, 803-814.

[3] B.A.Aliyev, Solvability of a boundary value problem with an operator in boundary conditions for a second order elliptic differential operator equation, DAN Azerb, SSR 37 (1981), no. 6, 17-21.

[4] V.V.Arestov, On exact inequliaties between the norms of functions and their derivatives, Acta sciente. Math. 33 (1972), no.364, 243-267.

[5] G.I.Aslanov, On solvability and asymptotic behavior of solutions of differential equaitons with operator coeficients in Hilbert space, Uspekhi mat. nauk issue 4 (1993), 172-173.

[6] V.I.Burenkov, On exact inequalities for the norms of intermediate derivatives on a finite interval, Trudy MIAN SSSR 173 (1986), 38-49.

[7] Yu.A.Dubinsky, Boundary value problems for some classes of arbitrary order differential-operator equaitons, DAN SSSR 196 (1971), no.1, 32-34.

[8] G.M.Eyvazly, On a unique solvability of a boundary value problems for a fourth order operator-differential equation in Hilbert space, Journal of Baku Engineering University. Mathematics and Computer Science 2 (2018), no. 2, 59-66.

[9] G.M. Eyvazly, On solvability of a boundary value problem for a fourth order homogeneous operator-differential equation in Hilbert space, Journal of Contemporary Applied Mathematics 8 (2018), no. 2, 2222-5498. 
[10] V.N.Gabushkin, On the best approximation of differentiation operator on a half straight, Matem. zametki issue 5 (1969) no. 6, 573-582.

[11] M.G.Gasymov, On the solvability of boundary-value problems for a class of operatordifferential equations, DAN SSSR 235 (1977), no.3, 505-508.

[12] G.T.Hardy, D.E.Littlwood, G.Polya, Inequalities. M. IL 1948, 456.

[13] A.N.Kolmogorov, On inequalities between upper bounds of successive derivatives of an arbitrary function on an infinite interval, Ucheniye zapiski MGU, Mathematika (1993), no. 3, 3-16.

[14] N.P.Kuptsov, Kolmogorov estimations for derivatives in the space $L_{2}[0, \infty]$, Trudy MIAN SSSR 138 (1975), 94-117.

[15] J.L.Lions, E.Magenes, Inhomogeneous boundary value problems and their applications, Moscow, Mir 1971, 371.

[16] Yu.I.Lyubich, On inequalities between the degrees of a linear operator, Izv. $A N$ SSSR, ser. math. 24 (1960), 825-864.

[17] S.S.Mirzoyev, On conditions of well-defined solvability of boundary value problems for operator-differential equations, DAN SSSR 273 (1983), no. 2, 292-296.

[18] S.S.Mirzoev, On the norms of operators of intermediate derivatives, 2003, Transactions of NASA XXIII (1998), no. 1, 157-165.

[19] A.A.Shkalikov, Elliptic equations in Hilbert space and related spectral problems, Proc. of I.G.Petrovsky workshop. Issue 14 (1989), 140-224.

[20] S.B.Stechkin, The best approximations of linear operators, Matem. zametki 1 (1967), no. 2, 137-149.

\section{Hamidulla I. Aslanov}

Institute of Mathematics and Mechanics of NAS of Azerbaijan, Baku, Azerbaijan

E-mail address: aslanov.50@mail.ru

Gunel M. Eyvazly

Sumgait State University, Sumgait, Azerbaijan

E-mail address: aliyevagunel193@mail.ru

Received: September 16, 2020; Revised: January 27, 2021; Accepted: February 11, 2021 